\title{
Análise de Interação Fluido-Estrutura na Asa de uma Aeronave Não Tripulada
}

\author{
Analysis of Fluid-Structure Interaction On the Wing of a Not Tripulated Aircraft
}

\section{Rimberg Antônio Tavares Júnior ${ }^{1}$ http://orcid.org/0000-0002-3040-8846}

\section{Eduardo César Miranda Loureiro ${ }^{1}$}

${ }^{1}$ Escola Politécnica de Pernambuco, Universidade de Pernambuco, Recife, Brasil.

E-mail do autor principal: Rimberg Antônio Tavares Júnior rimbergjr@gmail.com

\section{Resumo}

Em diversos campos da ciência e da engenharia encontramos problemas de interação entre fluido e estrutura, sendo necessário analisar tais fenômenos com a finalidade de prever e evitar danos estruturais. Uma análise numérica do fluxo de fluido sobre a asa de uma aeronave não tripulada, acoplada a uma análise da sua resposta estrutural nos permite identificar parâmetros de projeto que precisam ser corretamente ajustados para que a aeronave não entre em colapso durante o voo. Este artigo avalia as tensões e deformações estruturais em uma asa retangular, causadas pelos efeitos das forças aerodinâmicas, por meio de um modelo de Fluidodinâmica Computacional (CFD) e Análise de Elementos Finitos (FEA) parcialmente acoplados. Análise efetuada com a utilização do software de elementos finitos ANSYS 16.2, considerando-se um escoamento turbulento e incompressível, a velocidades subsônicas, em regime estacionário.

Palavras-Chave: Interação Fluido-Estrutura; Asa; Aeronave; Fluidodinâmica Computacional; Análise de Elementos Finitos.

\begin{abstract}
In several fields of science and engineering we find problems of interaction between fluid and structure, and it is necessary to analyze such phenomena in order to predict and avoid structural damages. A numerical fluid flow analysis on the wing of an unmanned aircraft coupled with an analysis of its structural response allows us to identify design parameters that need to be properly adjusted so that the aircraft does not collapse during flight. This paper intends to evaluate the structural deformations in a rectangular wing caused by the effects of aerodynamic forces, using a partially coupled Computational Fluid Dynamics (CFD) and Finite Element Analysis (FEA) model. ANSYS finite element software analysis, considering a turbulent and incompressible flow, at subsonic speeds, in steady state.
\end{abstract}

Key-words: Fluid-Structure Interaction, Wing, Aircraft, Computacional Fluid Dynamics, Finite Element Analysis. 


\section{Introdução}

Durante o projeto de um equipamento ou estrutura, cabe ao engenheiro verificar as tensões e deformações que a estrutura sofrerá devido às forças que estarão atuando sobre ela. Em diversos campos científicos e na engenharia, encontram-se exemplos de fenômenos que levam em consideração a interação entre fluido e estrutura, onde a deformação de um determinado corpo sólido passa a depender das forças exercidas pelo fluido que atua sobre ele. Dentro desse contexto podem-se citar as pás de uma turbina eólica, pás de uma turbina hidráulica ou a asa de um avião. Quando a geometria do corpo sólido varia devido a sua deformação, o escoamento do fluido sobre o corpo é modificado e isso altera as forças fluidodinâmicas atuantes sobre ele, gerando um ciclo que só acaba quando as forças internas da estrutura entram em equilíbrio com as forças externas devido à influência do fluido. Caso esta condição de equilíbrio não ocorra, as respostas seguirão para um padrão de instabilidade dinâmica, podendo causar danos na estrutura ou até mesmo sua falha catastrófica [1].

Os problemas desta natureza possuem soluções complexas e difíceis de encontrar analiticamente, sendo necessária a adoção de hipóteses simplificadoras para alcançar a solução desejada, o que não pode ser aplicado para problemas de difícil representação. Dentro deste contexto, surgem os métodos numéricos, que embora não forneçam soluções exatas, permitem analisar problemas complexos obtendo possíveis respostas, tomando como base valores numéricos para as variáveis em estudo com resultados próximos aos reais.

Analisar experimentalmente os parâmetros estruturais e aerodinâmicos que influenciam o comportamento da asa de uma aeronave durante o voo pode ser bastante custoso e complexo, tendo em vista que necessita de testes em túnel de vento com protótipos da aeronave, o que requer grande infraestrutura. A análise numérica aplicada à dinâmica dos fluidos, acoplada a códigos computacionais que verifiquem o comportamento da estrutura, podem fornecer previsões relevantes no tocante ao escoamento do ar sobre uma aeronave, analisando a influencia da distribuição de pressão e das forças atuantes nas tensões e deformações do corpo. O objetivo deste trabalho é simular as tensões e deformações na estrutura da asa de uma aeronave não tripulada pela influência de um campo de pressão gerado por um escoamento turbulento e incompressível a uma velocidade subsônica.

\section{Revisão Bibliográfica}

\subsection{Aeroelasticidade}

Os problemas de aeroelasticidade tratam da interação do ar e uma determinada estrutura, os quais foram descritos por Collar [2] e relacionam forças inerciais, elásticas e aerodinâmicas. As forças aerodinâmicas são resultantes do escoamento, estudadas pelos conceitos de mecânica dos fluidos; as forças elásticas referem-se aos problemas de mecânica dos sólidos ou resistência dos materiais; e as forças inerciais estão atreladas a problemas dinâmicos que ocorrem com as estruturas [3]. A associação de forças elásticas e inerciais geram problemas de vibrações mecânicas. A combinação de forças aerodinâmicas e inerciais configuram caso de estabilidade dinâmica, importantes no dimensionamento de componentes estruturais relacionados à sustentação de uma aeronave. Problemas envolvendo forças inerciais e aerodinâmicas configuram caso de aeroelasticidade estática, também chamados de problemas estáticos de interação fluido-estrutura, os quais ocorrem quando a movimentação do sólido é pouca ou nula. Por fim, situações que configuram aeroelasticidade dinâmica ocorrem quando temos a combinação das três forças envolvidas [3]. O Diagrama de Collar, exemplificado na Figura 1, descreve a interação entre estas forças.

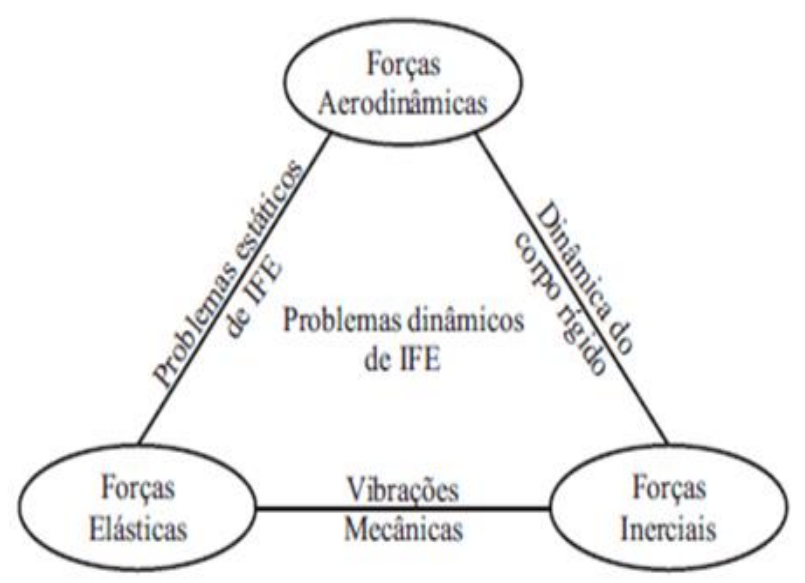

Figura 1: Diagrama de Collar. Fonte: [3]. 


\subsection{Método dos Elementos Finitos}

Segundo Bathe [4], o método dos elementos finitos (MEF) é um método numérico empregado na resolução de problemas físicos em análise de projetos de engenharia, aproximando as soluções de equações diferenciais parciais de qualquer campo da matemática avançada. De acordo com Narasaiah [5], em uma análise de elementos finitos (FEA) a estrutura é representada por um modelo mais simples, composta por um número finito de elementos conectados por pontos comuns, denominados como nós. Ao aplicar uma determinada solicitação na estrutura, avaliam-se as variáveis desejadas em cada elemento em particular, para depois verificar o resultado da estrutura como um todo, a qual está representada pela associação desses elementos.

De acordo com Bathe [4], por ser um procedimento numérico, faz-se necessário verificar a precisão da solução através da repetição da análise com parâmetros mais aguçados. Geralmente, aumenta-se o número de elementos e nós que representam a estrutura, refinando a malha gerada para verificar a variação das respostas obtidas. Em uma análise de fluidodinâmica computacional (CFD), o método utilizado é o de volumes finitos, que considera um volume de controle para solucionar balanços de massa, energia e quantidade de movimento [6].

Os problemas de interação fluido-estrutura (FSI) integram estes dois campos de análise, o de CFD, que utiliza um sistema fixo de coordenadas espacial, ou euleriano, e o de FEA, que usa um sistema fixo de coordenadas material, ou lagrangeano. Para resolver o problema introduz-se um algoritmo de acoplamento entre as duas formulações, chamado de método Lagrangeano-Euleriano Arbitrário (ALE). Os métodos para resolver o acoplamento das equações de FSI são classificados como: monolítico (não particionado), de acoplamento forte; e sequencial (particionado), que pode ser de acoplamento forte (com a utilização de sub-iterações para a sincronização dos campos físicos) ou fraco (sem sincronização entre os campos físicos) [1].

O software ANSYS 2013 apresenta uma outra classificação para análises de FSI: unidirecionais (1way), onde apenas uma modelagem física influencia a outra e os dados são transferidos em apenas uma direção; e bidirecionais (2-way), em que as duas modelagens físicas, CFD e FEA, são resolvidas em conjunto e trocam informações entre si continuamente. Naturalmente, o modelo bidirecional é mais rigoroso no sentido físico, pois o deslocamento estrutural responde de forma instantânea ao escoamento do fluido, consequentemente alterando a forma do escoamento até alcançar o equilíbrio, sendo mais utilizado para análises de aeroelasticidade dinâmica.

\subsection{Equações Governamentais}

\subsubsection{Equações de Navier-Stokes}

O movimento de qualquer partícula fluida é descrito pelas equações de Navier-Stokes, um conjunto de equações diferenciais acopladas definidas pela associação das equações de conservação de massa, conservação da quantidade de movimento e conservação de energia, e que descrevem como a pressão, a temperatura, e a densidade de um fluido estão relacionadas entre si. As equações de Navier-Stokes estão descritas nas equações (1) a (3), considerando um fluido em meio contínuo e de comportamento newtoniano:

$$
\begin{aligned}
& \rho\left(\frac{\partial u}{\partial t}+u \frac{\partial u}{\partial x}+v \frac{\partial u}{\partial y}+w \frac{\partial u}{\partial z}\right)=\rho g_{x}-\frac{\partial p}{\partial x}+\mu\left(\frac{\partial^{2} u}{\partial x^{2}}+\frac{\partial^{2} u}{\partial y^{2}}+\frac{\partial^{2} u}{\partial z^{2}}\right) \\
& \rho\left(\frac{\partial v}{\partial t}+u \frac{\partial v}{\partial x}+v \frac{\partial v}{\partial y}+w \frac{\partial v}{\partial z}\right)=\rho g_{\gamma}-\frac{\partial p}{\partial y}+\mu\left(\frac{\partial^{2} v}{\partial x^{2}}+\frac{\partial^{2} v}{\partial y^{2}}+\frac{\partial^{2} v}{\partial z^{2}}\right) \\
& \rho\left(\frac{\partial w}{\partial t}+u \frac{\partial w}{\partial x}+v \frac{\partial w}{\partial y}+w \frac{\partial w}{\partial z}\right)=\rho g_{z}-\frac{\partial p}{\partial z}+\mu\left(\frac{\partial^{2} w}{\partial x^{2}}+\frac{\partial^{2} w}{\partial y^{2}}+\frac{\partial^{2} w}{\partial z^{2}}\right)
\end{aligned}
$$

Onde, em notação vetorial, temos que: $\rho \frac{D \vec{V}}{D t}$ representa os termos convectivos de transporte (massa por unidade de volume vezes aceleração); $\rho \vec{g}$ é a força de campo; $\nabla p$ é a força de superfície; e $\mu \nabla^{2} \vec{V}$ é o termo de difusão (forças viscosas por unidade de volume).

Segundo Bordin [1], uma das formas de solucionar problemas de escoamento turbulento através de simulação numérica é pelas equações de NavierStokes em Médias de Reynolds (RANS), que são obtidas ao se decompor as variáveis como a soma de valores médios mais flutuações, com a média das flutuações igual a zero. Com isso, aparece um termo adicional chamado de tensor de Reynolds, que estabelece uma correlação entre as flutuações de massa específica e velocidade. Na equação (4), a RANS está representada em forma matricial,

http: / / dx.doi.org/10.25286/repa.v3i2.549 
desprezando as forças de campo, e na equação (5) pode-se observar o tensor de Reynolds.

$$
\begin{aligned}
& \frac{\partial \rho \overline{\mathrm{u}}_{\iota}}{\partial \mathrm{t}}+\frac{\partial}{\partial x_{j}} \rho\left(\bar{u}_{j} \bar{u}_{i}\right)=-\frac{\partial \overline{\mathrm{p}}}{\partial x_{i}}+\frac{\partial}{\partial x_{j}}\left(\mu\left(\frac{\partial \bar{u}_{i}}{\partial x_{j}}+\frac{\overline{\partial u_{j}}}{\partial x_{i}}\right)-\rho \overline{u_{\prime}^{\prime} u_{\iota}^{\prime}}\right) \\
& T_{i j}=-\rho \overline{u_{\jmath}^{\prime} u_{\iota}^{\prime}}=-\mu_{t}\left(\frac{\partial \bar{u}_{i}}{\partial x_{j}}+\frac{\overline{\partial u_{j}}}{\partial x_{i}}\right)-\frac{2}{3} k \delta_{i j}
\end{aligned}
$$

Onde: $\overline{u_{j}^{\prime} u_{l}^{\prime}}$ é a média do produto das flutuações; $T_{i j}$ é o tensor de Reynolds; $\mu_{t}$ é a viscosidade turbulenta; $k$ é a energia cinética turbulenta; e $\delta_{i j}$ é o delta de Kronecker, dado pela equação (6) [3].

$$
\delta_{i j}= \begin{cases}0 ; & i \neq j \\ 1 ; & i=j\end{cases}
$$

\subsubsection{Modelo de Turbulência k-w SST}

Segundo Freire et al. [7], os problemas de escoamento turbulento só podem ser resolvidos quando se tem um novo conjunto de equações que relacione as grandezas médias às grandezas instantâneas, daí surgem os modelos de turbulência. Os modelos de duas equações são mais utilizados para aplicações de CFD, pois são compostos de uma equação de transporte para a energia cinética turbulenta e de outra para o comprimento característico ou para a dissipação da energia cinética turbulenta. Segundo Moura et al. [8], os modelos mais empregados são: o k- $\omega$, de equações de transporte em função da energia cinética turbulenta (k) e da taxa de dissipação específica $(\omega)$, e $\circ k-\epsilon$, em função da energia cinética turbulenta $(k)$ e da taxa de dissipação da energia cinética turbulenta $(\epsilon)$. Próximo à camada limite, o modelo k$\omega$ é mais preciso que ok- $\epsilon$, pois é mais sensível às condições de contorno de turbulência em escoamento livre. O modelo de transporte de tensão cisalhante, k- $\omega$ SST, combina os outros dois citados anteriormente, selecionando o modelo $\mathrm{k}-\omega \mathrm{em}$ regiões próximas da parede, mudando a seleção para o modelo k- $\epsilon$ no escoamento livre.

\section{Metodologia}

\subsection{Configuração do Problema}

Para analisar as tensões e deformações estruturais que a asa de uma determinada aeronave sofre devido à atuação de um escoamento turbulento utilizou-se um algoritmo do tipo unidirecional, tratando a análise fluidodinâmica em separado da análise estrutural e depois acoplou-se os dois módulos através de uma interface fluido-estrutura. A análise das forças aerodinâmicas e suas influências foram realizadas através de uma modelagem tridimensional com o modelo de turbulência k- $\omega$ SST, desenvolvido para a simulação de escoamentos aerodinâmicos externos. Uma vez que as soluções de campo de fluxo convergiram, verificou-se o movimento de flexão causado pela influência dessas forças na asa, que estará em regime estático, engastada em uma de suas extremidades e livre para se deslocar na outra. O algoritmo para a análise proposta está exemplificado na Figura 2.

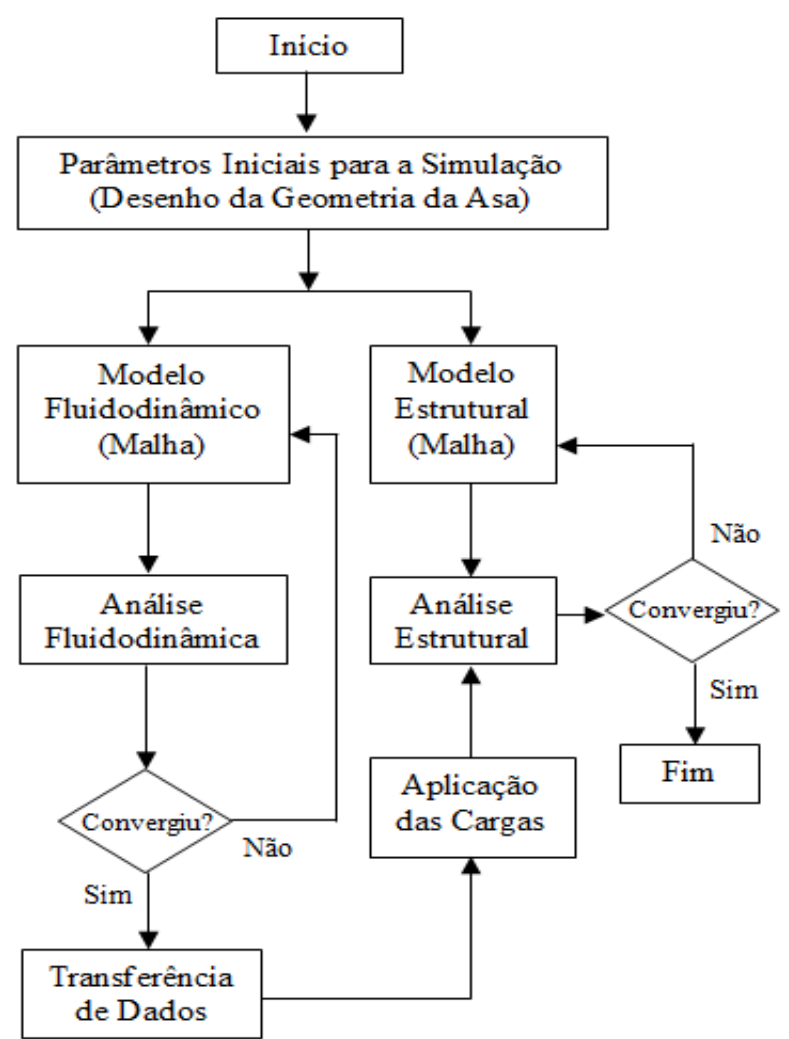

Figura 2: Algoritmo para Simulação de Interação FluidoEstrutura Parcialmente Acoplada.

Fonte: Adaptado de [9]. 
A simulação foi realizada no software de elementos finitos ANSYS 16.2, utilizando o Fluent para a análise de CFD e logo após transferidos os dados obtidos na solução fluidodinâmica para o Static Structural. Com o campo de pressão gerado pelo escoamento turbulento aplicado sobre o corpo da asa, pode-se então verificar as tensões e deformações geradas na estrutura.

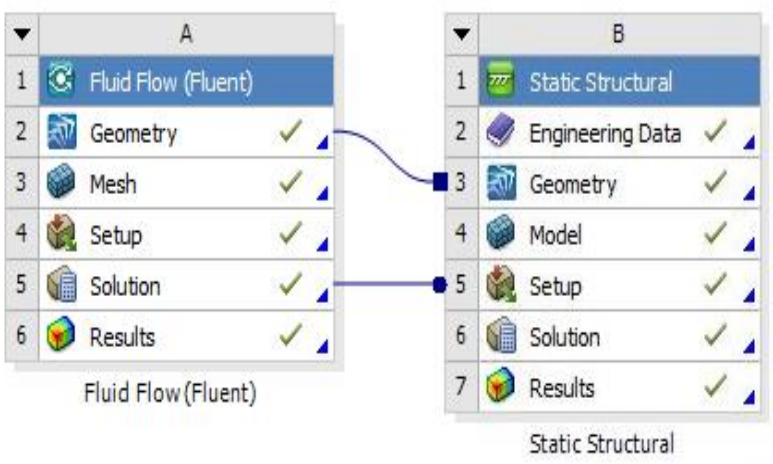

Figura 3: Projeto Esquemático de uma Análise de FSI na Plataforma ANSYS Workbench.

Fonte: Autor (2016).

\subsection{Descrição do Modelo}

A asa escolhida para as simulações compõe a aeronave projetada pelos alunos da Universidade de Pernambuco, componentes da equipe Tenpest Aerodesign UPE, para participar da Competição SAE Brasil Aerodesign, que ocorreu em novembro de 2016, em São José dos Campos, São Paulo. Detalhes da competição podem ser encontrados em SAE Brasil Aerodesign $\AA$ (2016). A aeronave pode ser vista na Figura 4.

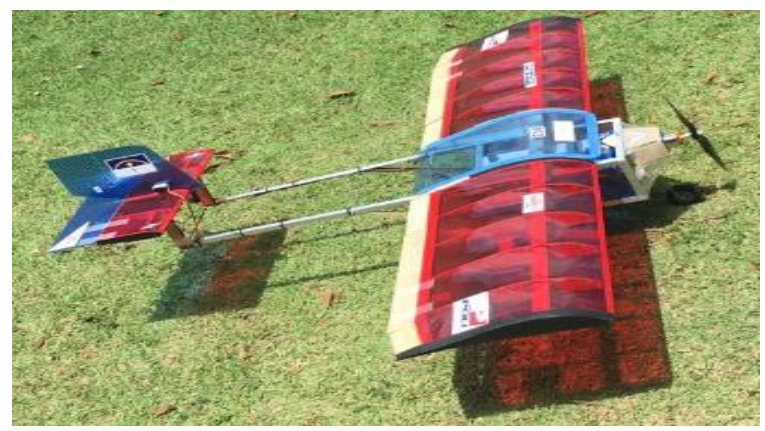

Figura 4: Aeronave da Tenpest Aerodesign 2016. Fonte: Autor (2016).
A asa possui uma geometria retangular e o aerofólio utilizado foi o Wortmann FX 74, conforme definido pelos parâmetros de projeto da Tenpest Aerodesign [10]. Para realizar a simulação considerou-se apenas metade da asa, pois, por haverem menos elementos no modelo, levará menos tempo para se encontrar a solução. A seção simulada possui uma envergadura de $650 \mathrm{~mm}$ e altura de 40 $\mathrm{mm}$ e levando em consideração sua simetria, os resultados obtidos poderão ser aplicados para a estrutura como um todo.

Levando em consideração que cada aerofólio que compõe a asa possui uma espessura muito menor do que seu comprimento, a estrutura foi modelada em elemento de casca, onde os perfis aerodinâmicos e a longarina (formada por uma alma e abas superior e inferior, configurando uma seção em formato de I), possuem $6 \mathrm{~mm}$ de espessura, com uma entelagem de espessura igual a $0,5 \mathrm{~mm}$. $O$ material utilizado para a asa foi a madeira balsa, cujas propriedades mecânicas estão listadas na Tabela 1.

Tabela 1: Propriedades Mecânicas da Asa.

\begin{tabular}{cc} 
Propriedade & Balsa \\
\hline Densidade, $\rho$ & $159,99 \mathrm{~kg} / \mathrm{m}^{3}$ \\
Módulo de Elasticidade, $\mathrm{E}$ & $2,55-3,17 \mathrm{GPa}$ \\
Coeficiente de Poisson, u & 0,29 \\
Tensão de Escoamento, $\sigma_{\mathrm{y}}$ & $20 \mathrm{MPa}$ \\
\hline
\end{tabular}

Fonte: [9].

O volume de controle utilizado para a análise fluidodinâmica possui um raio de $4 \mathrm{~m}$, junto a uma superfície retangular de comprimento igual a $6 \mathrm{~m}$ com uma profundidade de $4 \mathrm{~m}$. O modelo da asa foi subtraído do volume de controle, permitindo que a face externa da sua geometria fique em contato com a face interna do volume de controle, representando a sua imersão no fluido, isso possibilitará a interpolação entre os nós das malhas de ambos os domínios para obter o acoplamento dos campos. 0 modelo está ilustrado nas figuras 5 e 6 . 


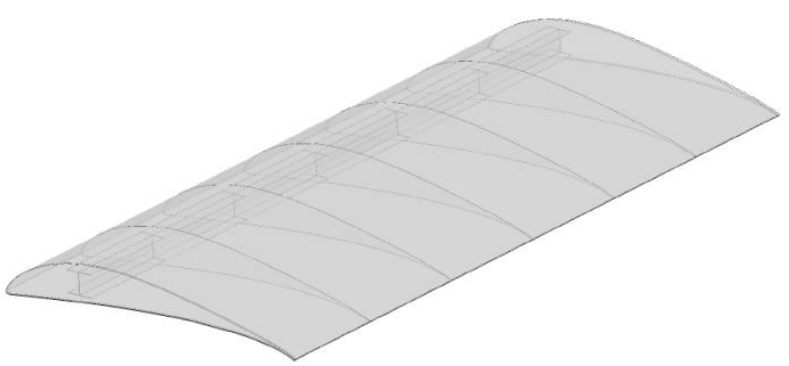

Figura 5: Modelagem da Asa.

Fonte: Autor (2016).

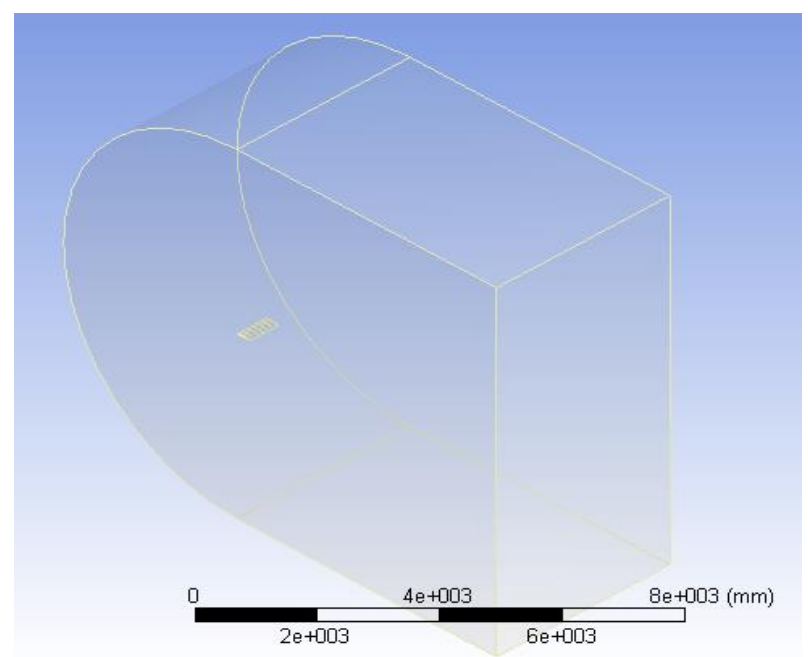

Figura 6: Volume de Controle com Asa Imersa. Fonte: Autor (2016).

\subsection{Geração da Malha}

A malha do volume de controle foi gerada com elementos tetraédricos para sólidos 3D definidos por 4 nós, cada nó possui 3 graus de liberdade: deslocamento em $x, y$ e $z$. O modelo possui uma malha mais refinada no centro, próximo à interface fluido/asa, para que possa representar bem os efeitos do escoamento. Ao todo, a malha do volume de controle possui 706.560 elementos interligados por 158.795 nós.

A estrutura foi modelada como elementos de casca tridimensionais triangulares, definidos por uma dimensão muito menor que as demais. Ao gerar a malha com este elemento é possível desenvolver um modelo de movimentação a partir da superfície média da estrutura, reduzindo o número de graus de liberdade do problema. Cada elemento utilizado para a malha do modelo estrutural possui 6 nós, cada um com 6 graus de liberdade: deslocamento e rotação em $x, y$ e $z$. A malha da asa possui 63.219 elementos, sendo 8.803 referentes à estrutura 6 interna da asa e 54.416 referentes à casca. Estes elementos estão conectados por 31.567 nós. As malhas geradas podem ser observadas nas figuras 7 , 8 e 9.

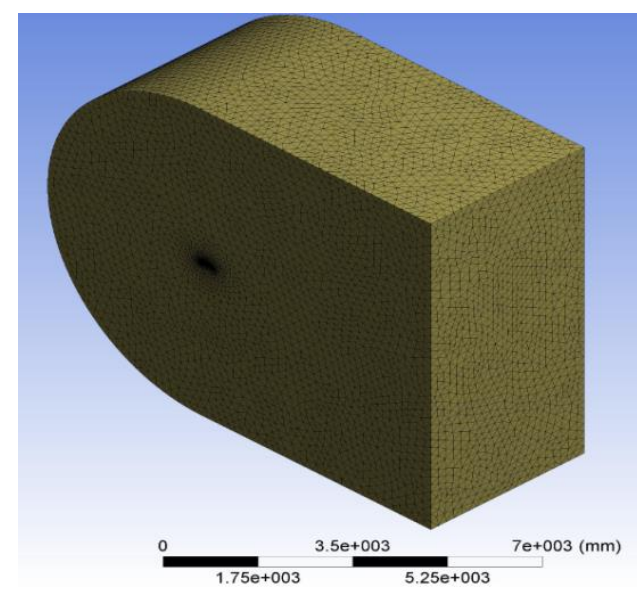

Figura 7: Volume de Controle com Asa Imersa. Fonte: Autor (2016).

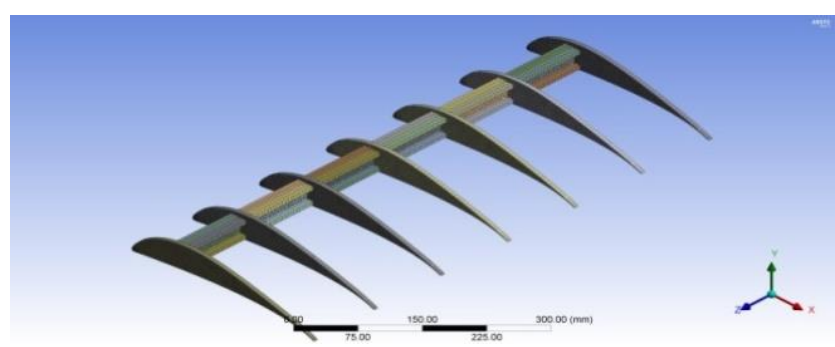

Figura 8: Malha da Estrutura Interna da Asa. Fonte: Autor (2016).

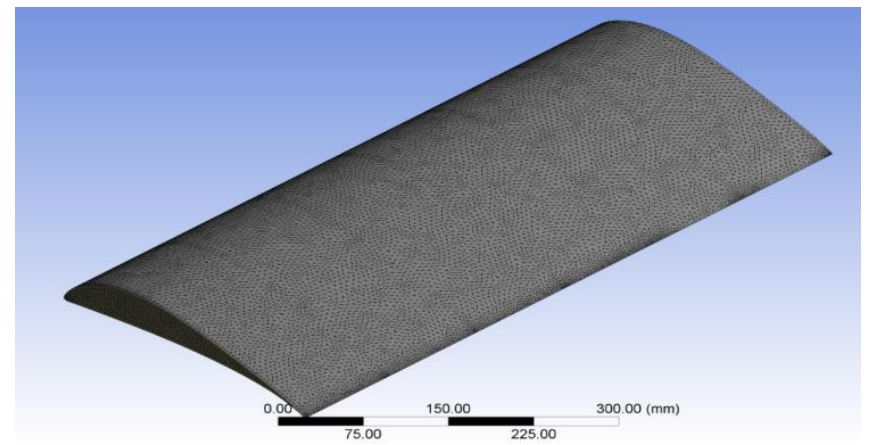

Figura 9: Malha da Casca Externa da Asa. Fonte: Autor (2016). 


\subsection{Condições de Contorno}

$\mathrm{Na}$ entrada do volume de controle considerou-se uma velocidade de $15 \mathrm{~m} / \mathrm{s}$, na direção normal à asa, considerando-a parada com o ar escoando livremente sobre a sua superfície. Este parâmetro de velocidade representa bem a condição real de voo, pois, de acordo com o Atlas do Potencial Eólico Brasileiro (2001), a velocidade média do vento na Região do Nordeste não passa de 9 m/s, em uma altura de até $50 \mathrm{~m}$.

A superfície da aeronave dentro do volume de controle é considerada como interface fluidoestrutura, e tanto o espaço vazio do meio fluido como as demais superfícies externas, são considerados como parede. O parâmetro de saída consiste em pressão igual a 0 . A estrutura foi considerada engastada em uma de suas extremidades, estando livre na outra. Uma representação do domínio com suas condições de contorno estão apresentados na Figura 10.

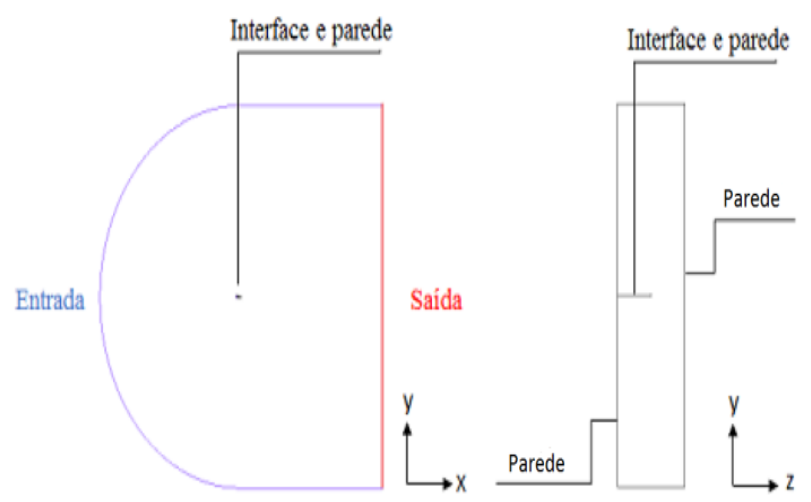

Figura 10: Malha da Estrutura Interna da Asa. Fonte: Adaptado de [1].

As propriedades do fluido e os valores de entrada referentes ao escoamento estão explicitados na Tabela 2. Tendo em vista que o número de Mach para a velocidade do escoamento turbulento é menor que 0,3 , ele será considerado incompressível.
Tabela 2: Propriedades do Volume de Controle.

\begin{tabular}{cc}
\hline Propriedade & Volume de Controle \\
\hline Fluido & $\mathrm{Ar}$ \\
\hline Densidade, $\rho$ & $1,225 \mathrm{~kg} / \mathrm{m}^{3}$ \\
Viscosidade, $\mu$ & $1,7894 \times 10^{-5} \mathrm{~kg} / \mathrm{m} . \mathrm{s}$ \\
Velocidade & $15 \mathrm{~m} / \mathrm{s}$ \\
Mach & 0,04412 \\
Reynolds & $3,08 \times 10^{5}$ \\
\hline
\end{tabular}

Fonte: Autor (2016).

\section{Resultados e Discussões}

Os resultados do escoamento em regime permanente com velocidade constante são levados em consideração para análise das forças atuantes sobre a estrutura da asa.

\subsection{Resultados do Escoamento Sobre a Asa}

$\mathrm{Na}$ análise fluidodinâmica, consideraram-se os campos de pressão e velocidade atuantes sobre o aerofólio, tanto na região da extremidade engastada como na região próximo à extremidade livre da asa. Foram analisadas ainda as distribuições de pressão estática em torno do modelo tridimensional da asa, o que pode ser verificado na figura 11 . Os parâmetros da variação de pressão são os mais importantes para a verificação da deformação estrutural.

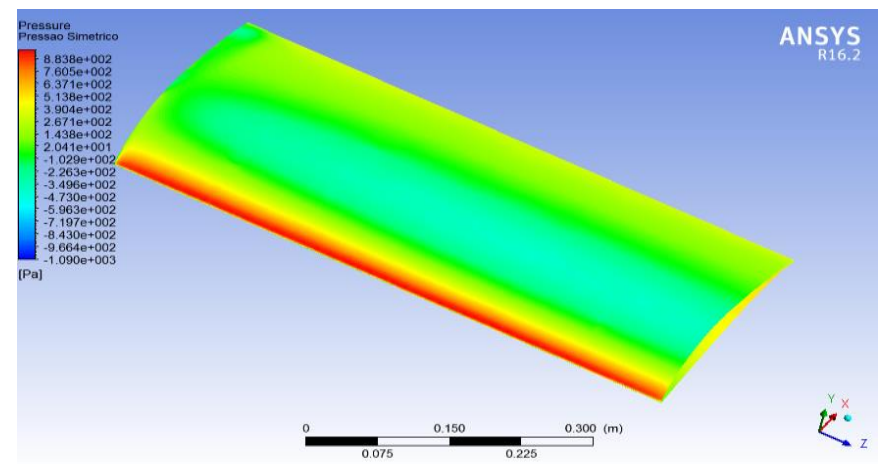

Figura 11: Distribuição de Pressão Sobre a Asa. Fonte: Autor (2016). 
De acordo com a imagem apresentada anteriormente, pode-se verificar que a influência da pressão máxima ocorre no bordo de ataque da asa, variando sua distribuição ao longo da superfície até chegar ao bordo de fuga. As figuras 12 a 15 mostram os campos de pressão e velocidade que atuam sobre o aerofólio, considerando-se ambas as extremidades, engastada e livre.

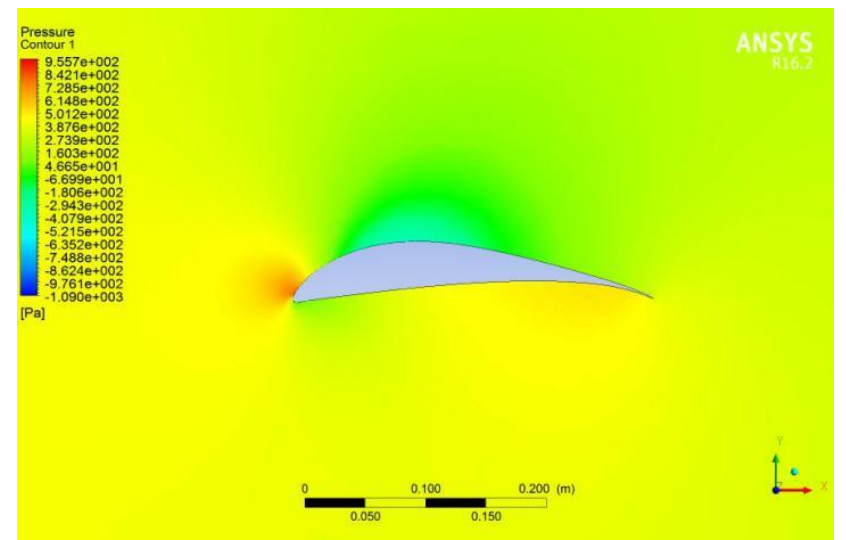

Figura 12: Campo de Pressão Sobre o Aerofólio na Extremidade Engastada.

Fonte: Autor (2016).

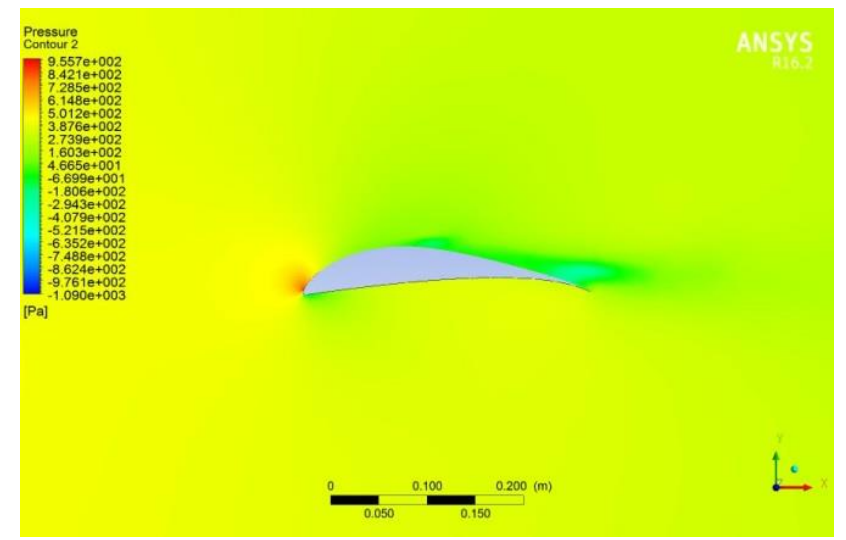

Figura 13: Campo de Pressão Sobre o Aerofólio Próximo à Extremidade Livre.

Fonte: Autor (2016).

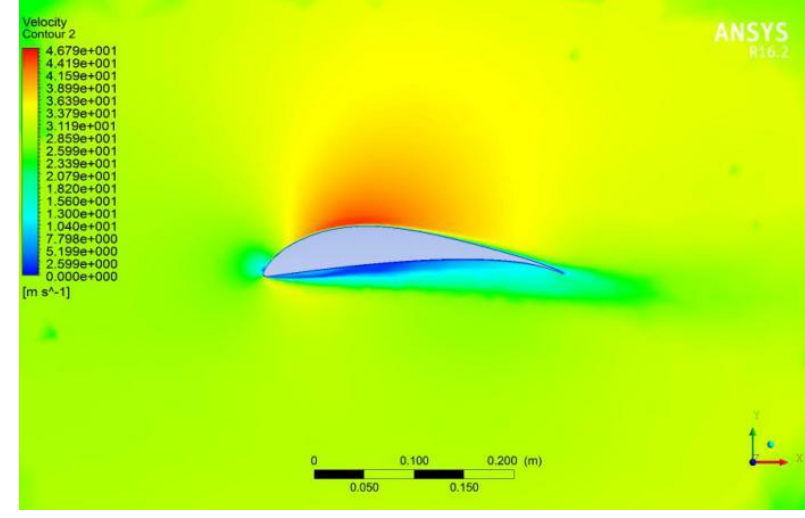

Figura 14: Campo de Velocidade Sobre o Aerofólio Próximo à Extremidade Engastada.

Fonte: Autor (2016).

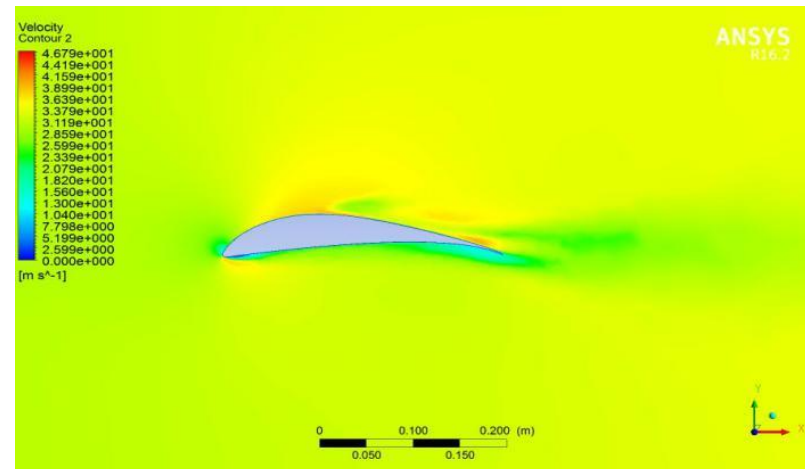

Figura 15: Campo de Velocidade Sobre o Aerofólio na Extremidade Livre.

Fonte: Autor (2016).

\subsection{Resultados da Análise Estrutural da Asa}

Nesta etapa importaram-se as tensões encontradas no resultado da análise fluidodinâmica para a simulação estrutural, aplicando-se a distribuição de pressão sobre a superfície da asa e verificando-se a deformação de sua estrutura. Para esta análise foram desconsiderados o amortecimento estrutural e a ação da gravidade. Os resultados podem ser verificados nas figuras 16 e 17 .

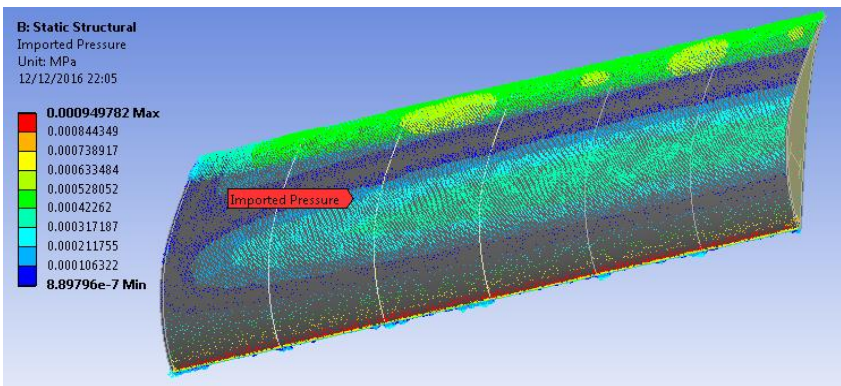

Figura 16: Distribuição da Pressão Sobre o Modelo Estrutural da Asa. 
Fonte: Autor (2016).

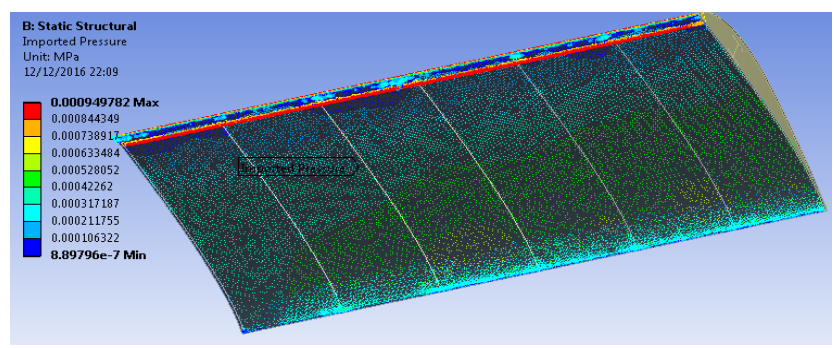

Figura 17: Pressão Máxima ao Longo do Bordo de Ataque.

Fonte: Autor (2016).

Pode-se verificar nas figuras 18 a 21 os contornos da deformação e tensão equivalente de von-Mises devido à pressão atuante na asa, onde é possível verificar a localização de seus valores máximos, próximo à extremidade engastada. O valor máximo da tensão equivalente de von-Mises é de aproximadamente 9,05 $\mathrm{MPa}$ e o valor máximo da deformação unitária é de aproximadamente 0,3\%.

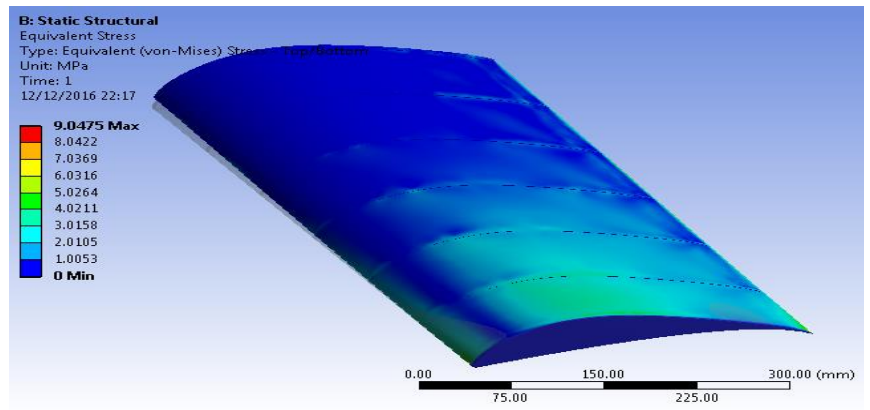

Figura 18: Tensão Máxima Equivalente de von-Mises na Estrutura.

Fonte: Autor (2016).

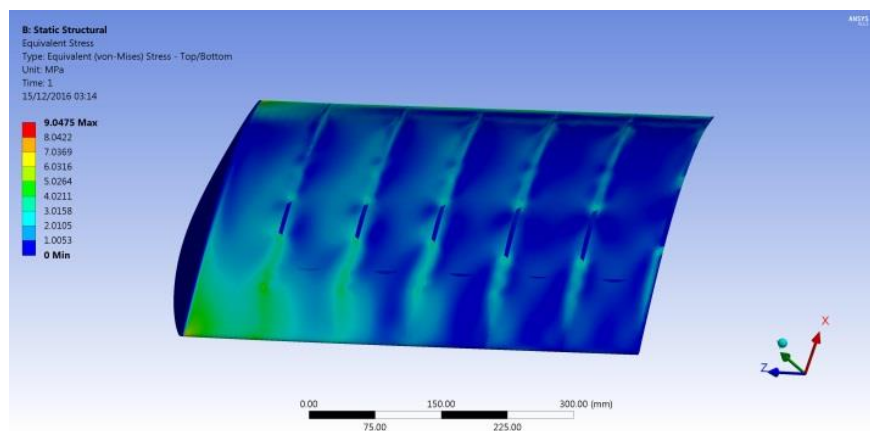

Figura 19: Tensão Máxima Equivalente na Parte Inferior da Asa.

Fonte: Autor (2016).

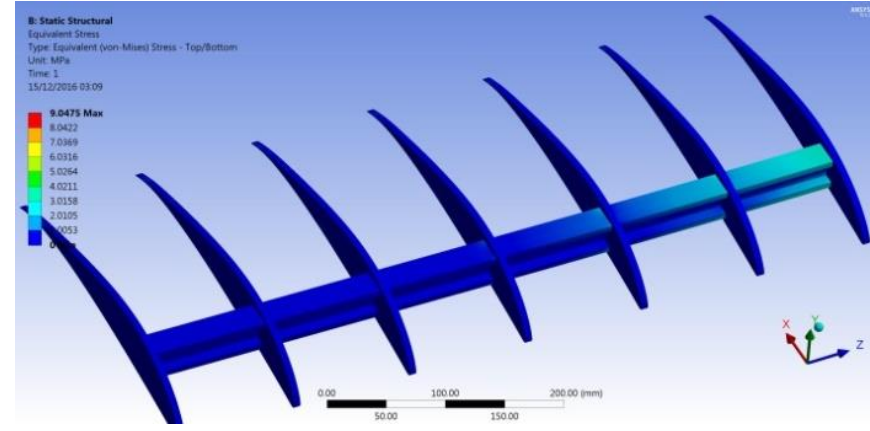

Figura 20: Detalhe da Tensão Atuante na Longarina. Fonte: Autor (2016).

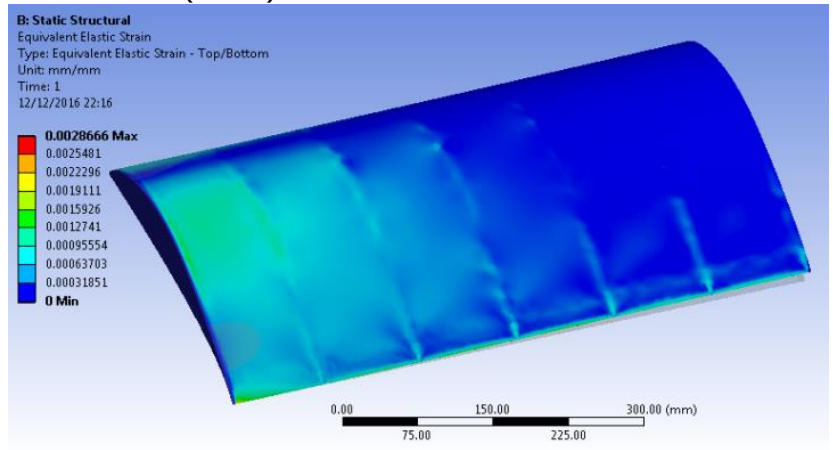

Figura 21: Deformação Máxima Equivalente de vonMises na Estrutura.

Fonte: Autor (2016).

A deformação total da asa devido à carga de pressão atuante sobre ela está reproduzida nas figuras 22 e 23. O valor da deformação aumenta gradualmente a partir do engaste até a extremidade livre. Pode-se perceber que a deformação máxima ocorre na extremidade livre da asa, principalmente na região do bordo de fuga, sendo de aproximadamente $6,88 \mathrm{~mm}$.

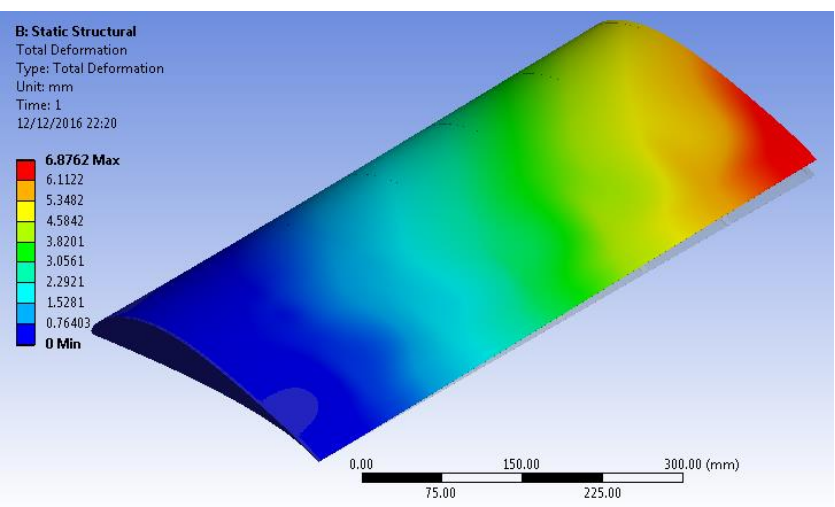

Figura 22: Deformação Total da Estrutura. Fonte: Autor (2016). 


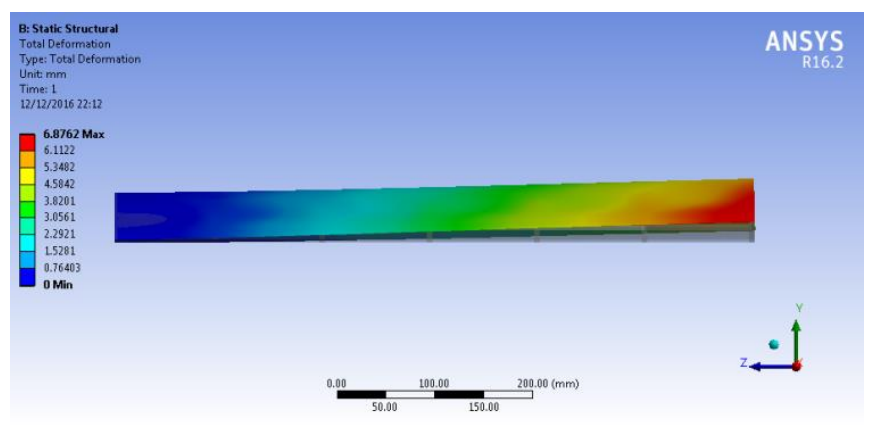

Figura 23: Detalhe do Deslocamento Máximo na Análise Estrutural.

Fonte: Autor (2016).

Pode-se verificar que a tensão máxima não ultrapassa a tensão de escoamento do material, não havendo plastificação ou risco de falha da estrutura da asa.

\section{Conclusões}

O trabalho em questão apresentou um estudo sobre análise de interação fluido-estrutura envolvendo o escoamento turbulento sobre meia asa de uma aeronave de pequeno porte, considerada engastada em uma extremidade e livre na outra. A proposta consiste em verificar a deformação da estrutura devido às forças fluidodinâmicas resultantes do escoamento turbulento. Para realizar a simulação computacional foi utilizado o software ANSYS 16.2, que através da plataforma Workbench acopla o Fluent, usado para a análise do escoamento, ao Static Structural, para a análise estrutural.

A simulação fluidodinâmica foi resolvida utilizando o método de volumes finitos baseado em RANS, com o modelo de turbulência k- $\omega$ SST. A análise estrutural foi realizada utilizando o método dos elementos finitos com um modelo em regime estático. Pôde-se identificar que a estrutura da asa está bem dimensionada para as condições impostas, tendo em vista que, devido às forças aplicadas em voo, as deformações estão dentro de limites aceitáveis.

Embora os experimentos físicos sejam a única forma de validação dos resultados, é possível a aplicação de estudos de convergência de malha para verificar a confiabilidade dos resultados obtidos na simulação, aumentando cada vez mais o número de elementos e nós até que os resultados não variem muito em relação aos valores obtidos nas iterações anteriores. Quanto mais nós e elementos a estrutura tiver, mais próximos da realidade serão os resultados. Sendo assim, pode-se concluir que a simulação numérica aplicada nos mais diversos campos da engenharia, bem como em situações de interação fluido-estrutura, nos dão uma excelente percepção da realidade, sendo uma alternativa mais acessível para verificar a integridade estrutural e aerodinâmica de uma aeronave não tripulada.

\section{Referências}

[1] BORDIN, Franciele Stail. Análise do Efeito da Interação Fluido-Estrutura nas Forças Fluidodinâmicas em um Elemento de Pá Flexível 3D. Dissertação de Mestrado. Programa de Pós-Graduação em Engenharia Mecânica da Universidade Federal do Rio Grande do Sul: RS, 2014. Disponível em: http://hdl.handle.net/10183/108499

[2] COLLAR, A. R. The Expanding Domain of Aeroelasticity. The Journal of the Royal Aeronautical Society. England, v. 5, n. 428, 1946. p. 613-636. Disponível em: https://www.cambridge.org/core/journals/aerona utical-journal/article/the-expanding-domain-ofaeroelasticity/489D68FBDE5D9801BB5615F4F40 5C069

[3] FERNANDES, Jeferson W. Dossa. Interação Fluido-Estrutura com Escoamentos Incompressíveis Utilizando o Método dos Elementos Finitos. Dissertação de Mestrado. Universidade de São Paulo, 2016.Disponível em: http://www.teses.usp.br/teses/disponiveis/18/18 134/tde-31032016-165546/pt-br.php

[4] BATHE, K. J. Finite Element Procedures. New Jersey: Prentice Hall, 1996.

[5] NARASAIAH, G. L. Finite Element Analysis. Hyderabad: BS Publications, 2008.

[6] SILVA JUNIOR, Luiz Justino. COSTA, Flávio Pietrobon. Simulação de Aerofólio NACA 0012 Utilizando Modelo de Turbulência k- $\epsilon$. In: Congresso Brasileiro de Engenharia de Produção, 5., 2015, Ponta Grossa. Anais... Ponta Grossa, PR: APREPO, dez. 2015. Disponível em: 
http://docplayer.com.br/49857694-Simulacaode-aerofolio-naca-0012-utilizando-modelo-deturbulencia-k-e-simulation-of-naca-0012-airfoilusing-k-e-turbulence-model.html

[7] FREIRE, A.; MENUT, P.; SU, J. Turbulência. Rio de Janeiro, Associação Brasileira de Ciências Mecânicas (ABCM), 2002.

[8] MOURA, Newton Reis de. Simulação Fluidodinâmica Computacional de Desempenho de um Impelidor de um Compressor Centrífugo. Dissertação de Mestrado. [229 p.] Programa de Pós Graduação da Universidade Federal do Rio de Janeiro: Rio de Janeiro, 2008. Disponível em: http://w2.files.scire.net.br/atrio/ufrjpem upl/THESIS/141/pemufrj2008mscnewtonrei sdemoura.pdf

[9] HUO, Shi-hui; YUAN, Zhe; WANG, Fusheng; YUE, Zhu-feng. Effects of Static Aeroelasticity on Composite Wing Characteristics Under Different Flight Attitudes. Jornal of Central South University, v.20, n.2, feb. 2013. Disponível https://link.springer.com/article/10.1007/s11771 $-013-1489-8$

[10] UNIVERSIDADE DE PERNAMBUCO. Tenpest Aerodesign In: Competição SAE Brasil Aerodesign, 18., 2002, Recife. Relatório de Projeto Estrutural. Universidade de Pernambuco: Recife, 2016.

[11] GOUD, T. Sai Kiran; SAI, Kumar A.; PRASAD, S. Srinivasa. Analysis of Fluid-Structure Interaction on an Aircraft Wing. International Journal of Engineering and Innovative Technology (IJEIT), v. 3, n. 9, China, mar. 2014. p.146-152. Disponível em: http://www.ijeit.com/Vol\%203/Issue\%209/IJEIT 1412201403 26.pdf

[12] BRUCE, Ralphin Rose J.; JINU, G. R.; MANIVEL, M.. Partly Coupled Fluid-Structure Interaction Analysis of an Aircraft Wing at Subsonics Speeds. International Journal of Mechanical \& Mechatronics Engineering, $v$. 14, n. 3, India, jun. 2014. p.22-29. Disponível em: http://www.ijens.org/Vol 14 I 03/1423035858-IJMME-IJENS.pdf
[13] BENINI, Gilherme Ribeiro. Modelo Numérico Para Simulação da Resposta Aeroelástica de Asas Fixas. Dissertação de Mestrado. [97 p.] Escola de Engenharia de São Carlos, Universidade de São Paulo. São Carlos: USP, $2002 . \quad$ Disponível em: http://www.teses.usp.br/teses/disponiveis/18/18 135/tde-14112002-193200/es.php

[14] SAE Brasil Aerodesign $®$ (2016) http://portal.saebrasil.org.br/programasestudantis/sae-brasil-aerodesign

[15] ANSYS. System Coupling User's Guide, 2013.

[16] Atlas do Potencial Eólico Brasileiro. Brasília, 2011. 